\title{
New Iterative Approximation Methods for a Countable Family of Nonexpansive Mappings in Banach Spaces
}

\author{
Kamonrat Nammanee ${ }^{1,2}$ and Rabian Wangkeeree ${ }^{2,3}$ \\ ${ }^{1}$ Department of Mathematics, School of Science and Technology, Phayao University, \\ Phayao 56000, Thailand \\ ${ }^{2}$ Centre of Excellence in Mathematics, CHE, Si Ayutthaya Road, Bangkok 10400, Thailand \\ ${ }^{3}$ Department of Mathematics, Faculty of Science, Naresuan University, Phitsanulok 65000, Thailand \\ Correspondence should be addressed to Rabian Wangkeeree, rabianw@nu.ac.th
}

Received 5 October 2010; Accepted 13 November 2010

Academic Editor: Qamrul Hasan Ansari

Copyright (C) 2011 K. Nammanee and R. Wangkeeree. This is an open access article distributed under the Creative Commons Attribution License, which permits unrestricted use, distribution, and reproduction in any medium, provided the original work is properly cited.

\begin{abstract}
We introduce new general iterative approximation methods for finding a common fixed point of a countable family of nonexpansive mappings. Strong convergence theorems are established in the framework of reflexive Banach spaces which admit a weakly continuous duality mapping. Finally, we apply our results to solve the the equilibrium problems and the problem of finding a zero of an accretive operator. The results presented in this paper mainly improve on the corresponding results reported by many others.
\end{abstract}

\section{Introduction}

In recent years, the existence of common fixed points for a finite family of nonexpansive mappings has been considered by many authors (see [1-4] and the references therein). The well-known convex feasibility problem reduces to finding a point in the intersection of the fixed point sets of a family of nonexpansive mappings (see $[5,6])$. The problem of finding an optimal point that minimizes a given cost function over the common set of fixed points of a family of nonexpansive mappings is of wide interdisciplinary interest and practical importance (see $[2,7])$. A simple algorithmic solution to the problem of minimizing a quadratic function over the common set of fixed points of a family of nonexpansive mappings is of extreme value in many applications including set theoretic signal estimation (see $[7,8])$. if

Let $E$ be a normed linear space. Recall that a mapping $T: E \rightarrow E$ is called nonexpansive

$$
\|T x-T y\| \leq\|x-y\|, \quad \forall x, y \in E .
$$


We use $F(T)$ to denote the set of fixed points of $T$, that is, $F(T)=\{x \in E: T x=x\}$. A self mapping $f: E \rightarrow E$ is a contraction on $E$ if there exists a constant $\alpha \in(0,1)$ such that

$$
\|f(x)-f(y)\| \leq \alpha\|x-y\|, \quad \forall x, y \in E .
$$

One classical way to study nonexpansive mappings is to use contractions to approximate a nonexpansive mapping [9-11]. More precisely, take $t \in(0,1)$ and define a contraction $T_{t}: E \rightarrow E$ by

$$
T_{t} x=t u+(1-t) T x, \quad \forall x \in E,
$$

where $u \in E$ is a fixed point. Banach's contraction mapping principle guarantees that $T_{t}$ has a unique fixed point $x_{t}$ in $E$. It is unclear, in general, what is the behavior of $x_{t}$ as $t \rightarrow 0$, even if $T$ has a fixed point. However, in the case of $T$ having a fixed point, Browder [9] proved that if $E$ is a Hilbert space, then $\left\{x_{t}\right\}$ converges strongly to a fixed point of $T$. Reich [10] extended Browder's result to the setting of Banach spaces and proved that if $E$ is a uniformly smooth Banach space, then $\left\{x_{t}\right\}$ converges strongly to a fixed point of $T$ and the limit defines the (unique) sunny nonexpansive retraction from $E$ onto $F(T)$. Xu [11] proved Reich's results hold in reflexive Banach spaces which have a weakly continuous duality mapping.

The iterative methods for nonexpansive mappings have recently been applied to solve convex minimization problems; see, for example, [12-14] and the references therein. Let $H$ be a real Hilbert space, whose inner product and norm are denoted by $\langle\cdot, \cdot\rangle$ and $\|\cdot\|$, respectively. Let $A$ be a strongly positive bounded linear operator on $H$; that is, there is a constant $\bar{\gamma}>0$ with property

$$
\langle A x, x\rangle \geq \bar{\gamma}\|x\|^{2}, \quad \forall x \in H .
$$

A typical problem is to minimize a quadratic function over the set of the fixed points of a nonexpansive mapping on a real Hilbert space $H$

$$
\min _{x \in F(T)} \frac{1}{2}\langle A x, x\rangle-\langle x, b\rangle,
$$

where $b$ is a given point in $H$. In 2003, Xu [13] proved that the sequence $\left\{x_{n}\right\}$ defined by the iterative method below, with the initial guess $x_{0} \in H$ chosen arbitrarily

$$
x_{n+1}=\left(I-\alpha_{n} A\right) T x_{n}+\alpha_{n} u, \quad n \geq 0
$$

converges strongly to the unique solution of the minimization problem (1.5) provided the sequence $\left\{\alpha_{n}\right\}$ satisfies certain conditions. Using the viscosity approximation method, Moudafi [15] introduced the following iterative process for nonexpansive mappings (see [16] for further developments in both Hilbert and Banach spaces). Let $f$ be a contraction on $H$. Starting with an arbitrary initial $x_{0} \in H$, define a sequence $\left\{x_{n}\right\}$ recursively by

$$
x_{n+1}=\left(1-\sigma_{n}\right) T x_{n}+\sigma_{n} f\left(x_{n}\right), \quad n \geq 0,
$$


where $\left\{\sigma_{n}\right\}$ is a sequence in $(0,1)$. It is proved $[15,16]$ that under certain appropriate conditions imposed on $\left\{\sigma_{n}\right\}$, the sequence $\left\{x_{n}\right\}$ generated by (1.7) strongly converges to the unique solution $x^{*}$ in $C$ of the variational inequality

$$
\left\langle(I-f) x^{*}, x-x^{*}\right\rangle \geq 0, \quad x \in H .
$$

Recently, Marino and $\mathrm{Xu}$ [17] mixed the iterative method (1.6) and the viscosity approximation method (1.7) and considered the following general iterative method:

$$
x_{n+1}=\left(I-\alpha_{n} A\right) T x_{n}+\alpha_{n} \gamma f\left(x_{n}\right), \quad n \geq 0,
$$

where $A$ is a strongly positive bounded linear operator on $H$. They proved that if the sequence $\left\{\alpha_{n}\right\}$ of parameters satisfies the following conditions:

(C1) $\lim _{n \rightarrow \infty} \alpha_{n}=0$,

(C2) $\sum_{n=1}^{\infty} \alpha_{n}=\infty$,

(C3) $\sum_{n=1}^{\infty}\left|\alpha_{n+1}-\alpha_{n}\right|<\infty$,

then the sequence $\left\{x_{n}\right\}$ generated by (1.9) converges strongly to the unique solution $x^{*}$ in $H$ of the variational inequality

$$
\left\langle(A-\gamma f) x^{*}, x-x^{*}\right\rangle \geq 0, \quad x \in H,
$$

which is the optimality condition for the minimization problem: $\min _{x \in C}(1 / 2)\langle A x, x\rangle-h(x)$, where $h$ is a potential function for $\gamma f$ (i.e., $h^{\prime}(x)=\gamma f(x)$ for $x \in H$ ).

On the other hand, in order to find a fixed point of nonexpansive mapping $T$, Halpern [18] was the first who introduced the following iteration scheme which was referred to as Halpern iteration in a Hilbert space: $x, x_{0} \in C,\left\{\alpha_{n}\right\} \subset[0,1]$,

$$
x_{n+1}=\alpha_{n} x+\left(1-\alpha_{n}\right) T x_{n}, \quad n \geq 0 .
$$

He pointed out that the control conditions (C1) $\lim _{n \rightarrow \infty} \alpha_{n}=0$ and (C2) $\sum_{n=1}^{\infty} \alpha_{n}=\infty$ are necessary for the convergence of the iteration scheme (1.11) to a fixed point of $T$. Furthermore, the modified version of Halpern iteration was investigated widely by many mathematicians. Recently, for the sequence of nonexpansive mappings $\left\{T_{n}\right\}_{n=1}^{\infty}$ with some special conditions, Aoyama et al. [1] studied the strong convergence of the following modified version of Halpern iteration for $x_{0}, x \in C$ :

$$
x_{n+1}=\alpha_{n} x+\left(1-\alpha_{n}\right) T_{n} x_{n}, \quad n \geq 0,
$$

where $C$ is a nonempty closed convex subset of a uniformly convex Banach space $E$ whose norm is uniformly Gáteaux differentiable, $\left\{\alpha_{n}\right\}$ is a sequence in $[0,1]$ satisfying (C1) $\lim _{n \rightarrow \infty} \alpha_{n}=0$, (C2) $\sum_{n=1}^{\infty} \alpha_{n}=\infty$, and either (C3) $\sum_{n=1}^{\infty}\left|\alpha_{n}-\alpha_{n+1}\right|<\infty$ or $\left(\mathrm{C} 3^{\prime}\right) \alpha_{n} \in(0,1]$ for all $n \in \mathbb{N}$ and $\lim _{n \rightarrow \infty}\left(\alpha_{n} / \alpha_{n+1}\right)=1$. Very recently, Song and Zheng [19] also introduced the conception of the condition $(B)$ on a countable family of nonexpansive mappings and proved 
strong convergence theorems of the modified Halpern iteration (1.12) and the sequence $\left\{y_{n}\right\}$ defined by

$$
y_{0}, y \in C, \quad y_{n+1}=T_{n}\left(\alpha_{n} y+\left(1-\alpha_{n}\right) y_{n}\right), \quad n \geq 0,
$$

in a reflexive Banach space $E$ with a weakly continuous duality mapping and in a reflexive strictly convex Banach space with a uniformly Gáteaux differentiable norm.

Other investigations of approximating common fixed points for a countable family of nonexpansive mappings can be found in [1,20-24] and many results not cited here.

In a Banach space $E$ having a weakly continuous duality mapping $J_{\varphi}$ with a gauge function $\varphi$, an operator $A$ is said to be strongly positive [25] if there exists a constant $\bar{\gamma}>0$ with the property

$$
\begin{gathered}
\left\langle A x, J_{\varphi}(x)\right\rangle \geq \bar{\gamma}\|x\| \varphi(\|x\|), \\
\|\alpha I-\beta A\|=\sup _{\|x\| \leq 1}\left|\left\langle(\alpha I-\beta A) x, J_{\varphi}(x)\right\rangle\right|, \quad \alpha \in[0,1], \beta \in[-1,1],
\end{gathered}
$$

where $I$ is the identity mapping. If $E:=H$ is a real Hilbert space, then the inequality (1.14) reduces to (1.4).

In this paper, motivated by Aoyama et al. [1], Song and Zheng [19], and Marino and $\mathrm{Xu}$ [17], we will combine the iterative method (1.12) with the viscosity approximation method (1.9) and consider the following three new general iterative methods in a reflexive Banach space $E$ which admits a weakly continuous duality mapping $J_{\varphi}$ with gauge $\varphi$ :

$$
\begin{gathered}
x_{0}=x \in E, \\
x_{n+1}=\alpha_{n} \gamma f\left(T_{n} x_{n}\right)+\left(I-\alpha_{n} A\right) T_{n} x_{n}, \quad n \geq 0, \\
z_{0}=z \in E, \\
z_{n+1}=\alpha_{n} \gamma f\left(z_{n}\right)+\left(I-\alpha_{n} A\right) T_{n} z_{n}, \quad n \geq 0, \\
y_{0}=y \in E, \\
y_{n+1}=T_{n}\left(\alpha_{n} \gamma f\left(y_{n}\right)+\left(I-\alpha_{n} A\right) y_{n}\right), \quad n \geq 0,
\end{gathered}
$$

where $A$ is strongly positive defined by (1.15), $\left\{T_{n}: E \rightarrow E\right\}$ is a countable family of nonexpansive mappings, and $f$ is an $\alpha$-contraction. We will prove in Section 3 that if the sequence $\left\{\alpha_{n}\right\}$ of parameters satisfies the appropriate conditions, then the sequences $\left\{x_{n}\right\},\left\{z_{n}\right\}$, and $\left\{y_{n}\right\}$ converge strongly to the unique solution $\tilde{x}$ of the variational inequality

$$
\left\langle(A-\gamma f) \tilde{x}, J_{\varphi}(\tilde{x}-p)\right\rangle \leq 0, \quad \forall p \in \bigcap_{n=1}^{\infty} F\left(T_{n}\right) .
$$

Finally, we apply our results to solve the the equilibrium problems and the problem of finding a zero of an accretive operator. 


\section{Preliminaries}

Throughout this paper, let $E$ be a real Banach space, and $E^{*}$ be its dual space. We write $x_{n} \rightarrow x$ (resp., $x_{n}-^{*} x$ ) to indicate that the sequence $\left\{x_{n}\right\}$ weakly (resp., weak*) converges to $x$; as usual $x_{n} \rightarrow x$ will symbolize strong convergence. Let $U=\{x \in E:\|x\|=1\}$. A Banach space $E$ is said to uniformly convex if, for any $\epsilon \in(0,2]$, there exists $\delta>0$ such that, for any $x, y \in U$, $\|x-y\| \geq \epsilon$ implies $\|(x+y) / 2\| \leq 1-\delta$. It is known that a uniformly convex Banach space is reflexive and strictly convex (see also [26]). A Banach space $E$ is said to be smooth if the limit $\lim _{t \rightarrow 0}((\|x+t y\|-\|x\|) / t)$ exists for all $x, y \in U$. It is also said to be uniformly smooth if the limit is attained uniformly for $x, y \in U$.

By a gauge function $\varphi$, we mean a continuous strictly increasing function $\varphi:[0, \infty) \rightarrow$ $[0, \infty)$ such that $\varphi(0)=0$ and $\varphi(t) \rightarrow \infty$ as $t \rightarrow \infty$. Let $E^{*}$ be the dual space of $E$. The duality mapping $J_{\varphi}: E \rightarrow 2^{E^{*}}$ associated to a gauge function $\varphi$ is defined by

$$
J_{\varphi}(x)=\left\{f^{*} \in E^{*}:\left\langle x, f^{*}\right\rangle=\|x\| \varphi(\|x\|), \quad\left\|f^{*}\right\|=\varphi(\|x\|)\right\}, \quad \forall x \in E .
$$

In particular, the duality mapping with the gauge function $\varphi(t)=t$, denoted by $J$, is referred to as the normalized duality mapping. Clearly, there holds the relation $J_{\varphi}(x)=$ $(\varphi(\|x\|) /\|x\|) J(x)$ for all $x \neq 0$ (see [27]). Browder [27] initiated the study of certain classes of nonlinear operators by means of the duality mapping $J_{\varphi}$. Following Browder [27], we say that a Banach space $E$ has a weakly continuous duality mapping if there exists a gauge $\varphi$ for which the duality mapping $J_{\varphi}(x)$ is single valued and continuous from the weak topology to the weak* topology, that is, for any $\left\{x_{n}\right\}$ with $x_{n} \rightarrow x$, the sequence $\left\{J_{\varphi}\left(x_{n}\right)\right\}$ converges weakly ${ }^{*}$ to $J_{\varphi}(x)$. It is known that $l^{p}$ has a weakly continuous duality mapping with a gauge function $\varphi(t)=t^{p-1}$ for all $1<p<\infty$. Set

$$
\Phi(t)=\int_{0}^{t} \varphi(\tau) d \tau, \quad \forall t \geq 0
$$

then

$$
J_{\varphi}(x)=\partial \Phi(\|x\|), \quad \forall x \in E,
$$

where $\partial$ denotes the subdifferential in the sense of convex analysis.

Now, we collect some useful lemmas for proving the convergence result of this paper.

The first part of the next lemma is an immediate consequence of the subdifferential inequality and the proof of the second part can be found in [28].

Lemma 2.1 (see [28]). Assume that a Banach space E has a weakly continuous duality mapping $J_{\varphi}$ with gauge $\varphi$.

(i) For all $x, y \in E$, the following inequality holds:

$$
\Phi(\|x+y\|) \leq \Phi(\|x\|)+\left\langle y, J_{\varphi}(x+y)\right\rangle .
$$


In particular, for all $x, y \in E$,

$$
\|x+y\|^{2} \leq\|x\|^{2}+2\langle y, J(x+y)\rangle
$$

(ii) Assume that a sequence $\left\{x_{n}\right\}$ in $E$ converges weakly to a point $x \in E$,

then the following identity holds:

$$
\limsup _{n \rightarrow \infty} \Phi\left(\left\|x_{n}-y\right\|\right)=\limsup _{n \rightarrow \infty} \Phi\left(\left\|x_{n}-x\right\|\right)+\Phi(\|y-x\|), \quad \forall x, y \in E
$$

Lemma 2.2 (see [1, Lemma 2.3]). Let $\left\{a_{n}\right\}$ be a sequence of nonnegative real numbers such that satisfying the property

$$
a_{n+1} \leq\left(1-\alpha_{n}\right) a_{n}+\alpha_{n} c_{n}+b_{n}, \quad \forall n \geq 0,
$$

where $\left\{\alpha_{n}\right\},\left\{b_{n}\right\},\left\{c_{n}\right\}$ satisfying the restrictions

(i) $\sum_{n=1}^{\infty} \alpha_{n}=\infty$; (ii) $\sum_{n=1}^{\infty} b_{n}<\infty$; (iii) $\lim \sup _{n \rightarrow \infty} c_{n} \leq 0$.

Then, $\lim _{n \rightarrow \infty} a_{n}=0$.

Definition 2.3 (see [1]). Let $\left\{T_{n}\right\}$ be a family of mappings from a subset $C$ of a Banach space $E$ into $E$ with $\bigcap_{n=1}^{\infty} F\left(T_{n}\right) \neq \emptyset$. We say that $\left\{T_{n}\right\}$ satisfies the AKTT-condition if for each bounded subset $B$ of $C$,

$$
\sum_{n=1}^{\infty} \sup _{z \in B}\left\|T_{n+1} z-T_{n} z\right\|<\infty
$$

Remark 2.4. The example of the sequence of mappings $\left\{T_{n}\right\}$ satisfying AKTT-condition is supported by Lemma 4.6.

Lemma 2.5 (see [1, Lemma 3.2]). Suppose that $\left\{T_{n}\right\}$ satisfies AKTT-condition, then, for each $y \in$ $C,\left\{T_{n} y\right\}$ converses strongly to a point in $C$. Moreover, let the mapping $T$ be defined by

$$
T y=\lim _{n \rightarrow \infty} T_{n} y, \quad \forall y \in C
$$

Then, for each bounded subset $B$ of $C, \lim _{n \rightarrow \infty} \sup _{z \in B}\left\|T z-T_{n} z\right\|=0$.

The next valuable lemma was proved by Wangkeeree et al. [25]. Here, we present the proof for the sake of completeness.

Lemma 2.6. Assume that a Banach space $E$ has a weakly continuous duality mapping $J_{\varphi}$ with gauge $\varphi$. Let $A$ be a strongly positive bounded linear operator on $E$ with coefficient $\bar{\gamma}>0$ and $0<\rho \leq$ $\varphi(1)\|A\|^{-1}$, then $\|I-\rho A\| \leq \varphi(1)(1-\rho \bar{\gamma})$. 
Proof. From (1.15), we obtain that $\|A\|=\sup _{\|x\| \leq 1}\left|\left\langle A x, J_{\varphi}(x)\right\rangle\right|$. Now, for any $x \in E$ with $\|x\|=1$, we see that

$$
\left\langle(I-\rho A) x, J_{\varphi}(x)\right\rangle=\varphi(1)-\rho\left\langle A x, J_{\varphi}(x)\right\rangle \geq \varphi(1)-\rho\|A\| \geq 0 .
$$

That is, $I-\rho A$ is positive. It follows that

$$
\begin{aligned}
\|I-\rho A\| & =\sup \left\{\left\langle(I-\rho A) x, J_{\varphi}(x)\right\rangle: x \in E,\|x\|=1\right\} \\
& =\sup \left\{\varphi(1)-\rho\left\langle A x, J_{\varphi}(x)\right\rangle: x \in E,\|x\|=1\right\} \\
& \leq \varphi(1)-\rho \bar{\gamma} \varphi(1)=\varphi(1)(1-\rho \bar{\gamma}) .
\end{aligned}
$$

Let $E$ be a Banach space which admits a weakly continuous duality $J_{\varphi}$ with gauge $\varphi$ such that $\varphi$ is invariant on $[0,1]$ that is, $\varphi([0,1]) \subset[0,1]$. Let $T: E \rightarrow E$ be a nonexpansive mapping, $t \in(0,1), f$ an $\alpha$-contraction, and $A$ a strongly positive bounded linear operator with coefficient $\bar{\gamma}>0$ and $0<\gamma<\bar{\gamma} \varphi(1) / \alpha$. Define the mapping $S_{t}: E \rightarrow E$ by

$$
S_{t}(x)=\operatorname{t\gamma } f(x)+(I-t A) T x, \quad \forall x \in E .
$$

Then, $S_{t}$ is a contraction mapping. Indeed, for any $x, y \in E$,

$$
\begin{aligned}
\left\|S_{t}(x)-S_{t}(y)\right\| & =\|\operatorname{tr}(f(x)-f(y))+(I-t A)(T x-T y)\| \\
& \leq \operatorname{tr}\|f(x)-f(y)\|+\|I-t A\|\|T x-T y\| \\
& \leq \operatorname{tr} \alpha\|x-y\|+\varphi(1)(1-t \bar{\gamma})\|x-y\| \\
& \leq[1-t(\varphi(1) \bar{\gamma}-\gamma \alpha)]\|x-y\| .
\end{aligned}
$$

Thus, by Banach contraction mapping principle, there exists a unique fixed point $x_{t}$ in $E$, that is

$$
x_{t}=\operatorname{tr} f\left(x_{t}\right)+(I-t A) T x_{t} .
$$

Remark 2.7. We note that $l^{p}$ space has a weakly continuous duality mapping with a gauge function $\varphi(t)=t^{p-1}$ for all $1<p<\infty$. This shows that $\varphi$ is invariant on $[0,1]$.

Lemma 2.8 (see [25, Lemma 3.3]). Let $E$ be a reflexive Banach space which admits a weakly continuous duality mapping $J_{\varphi}$ with gauge $\varphi$ such that $\varphi$ is invariant on $[0,1]$. Let $T: E \rightarrow E$ be a nonexpansive mapping with $F(T) \neq \emptyset, f$ an $\alpha$-contraction, and $A$ a strongly positive bounded linear operator with coefficient $\bar{\gamma}>0$ and $0<\gamma<\bar{\gamma} \varphi(1) / \alpha$. Then, the net $\left\{x_{t}\right\}$ defined by (2.14) converges strongly as $t \rightarrow 0$ to a fixed point $\tilde{x}$ of $T$ which solves the variational inequality

$$
\left\langle(A-\gamma f) \tilde{x}, J_{\varphi}(\tilde{x}-p)\right\rangle \leq 0, \quad p \in F(T) .
$$




\section{Main Results}

We now state and prove the main theorems of this section.

Theorem 3.1. Let $E$ be a reflexive Banach space which admits a weakly continuous duality mapping $J_{\varphi}$ with gauge $\varphi$ such that $\varphi$ is invariant on $[0,1]$. Let $\left\{T_{n}: E \rightarrow E\right\}_{n=0}^{\infty}$ be a countable family of nonexpansive mappings satisfying $F:=\bigcap_{n=0}^{\infty} F\left(T_{n}\right) \neq \emptyset$. Let $f$ be an $\alpha$-contraction and $A$ a strongly positive bounded linear operator with coefficient $\bar{\gamma}>0$ and $0<\gamma<\bar{\gamma} \varphi(1) / \alpha$. Let the sequence $\left\{x_{n}\right\}$ be generated by (1.16), where $\left\{\alpha_{n}\right\}$ is a sequence in $[0,1]$ satisfying the following conditions:

(C1) $\lim _{n \rightarrow \infty} \alpha_{n}=0$,

(C2) $\sum_{n=0}^{\infty} \alpha_{n}=\infty$,

(C3) $\sum_{n=0}^{\infty}\left|\alpha_{n}-\alpha_{n+1}\right|<\infty$.

Suppose that $\left\{T_{n}\right\}$ satisfies the AKTT-condition. Let $T$ be a mapping of $E$ into itself defined by $T z=$ $\lim _{n \rightarrow \infty} T_{n} z$ for all $z \in E$, and suppose that $F(T)=\bigcap_{n=0}^{\infty} F\left(T_{n}\right)$. Then, $\left\{x_{n}\right\}$ converges strongly to $\tilde{x}$ which solves the variational inequality

$$
\left\langle(A-\gamma f) \tilde{x}, J_{\varphi}(\tilde{x}-p)\right\rangle \leq 0, \quad \forall p \in F
$$

Proof. Applying Lemma 2.8, there exists a point $\tilde{x} \in F(T)$ which solves the variational inequality (3.1). Next, we observe that $\left\{x_{n}\right\}$ is bounded. Indeed, pick any $p \in F$ to obtain

$$
\begin{aligned}
\left\|x_{n+1}-p\right\| & =\left\|\alpha_{n} \gamma f\left(T_{n} x_{n}\right)+\left(I-\alpha_{n} A\right) T_{n} x_{n}-p\right\| \\
& =\left\|\alpha_{n}\left(\gamma f\left(T_{n} x_{n}\right)-A(p)\right)+\left(I-\alpha_{n} A\right) T_{n} x_{n}-\left(I-\alpha_{n} A\right) p\right\| \\
& =\left\|I-\alpha_{n} A\right\|\left\|T_{n} x_{n}-T_{n} p\right\|+\alpha_{n}\left\|\gamma f\left(T_{n} x_{n}\right)-A(p)\right\| \\
& \leq \varphi(1)\left(1-\alpha_{n} \bar{\gamma}\right)\left\|x_{n}-p\right\|+\alpha_{n} \gamma \alpha\left\|x_{n}-p\right\|+\alpha_{n}\|\gamma f(p)-A p\| \\
& \leq\left(\varphi(1)-\alpha_{n}(\varphi(1) \bar{\gamma}-\gamma \alpha)\right)\left\|x_{n}-p\right\|+\alpha_{n}\|\gamma f(p)-A(p)\| \\
& \leq\left(1-\alpha_{n}(\varphi(1) \bar{\gamma}-\gamma \alpha)\right)\left\|x_{n}-p\right\|+\alpha_{n}(\varphi(1) \bar{\gamma}-\gamma \alpha) \frac{\|\gamma f(p)-A(p)\|}{\varphi(1) \bar{\gamma}-\gamma \alpha} .
\end{aligned}
$$

It follows from induction that

$$
\left\|x_{n+1}-p\right\| \leq \max \left\{\left\|x_{0}-p\right\|, \frac{\|\gamma f(p)-A(p)\|}{\varphi(1) \bar{\gamma}-\gamma \alpha}\right\}, \quad n \geq 0
$$

Thus, $\left\{x_{n}\right\}$ is bounded, and hence so are $\left\{A T_{n} x_{n}\right\}$ and $\left\{f\left(T_{n} x_{n}\right)\right\}$. Now, we show that

$$
\lim _{n \rightarrow \infty}\left\|x_{n+1}-x_{n}\right\|=0
$$


We observe that

$$
\begin{aligned}
\left\|x_{n+1}-x_{n}\right\|= & \left\|\alpha_{n} \gamma f\left(T_{n} x_{n}\right)+\left(I-\alpha_{n} A\right) T_{n} x_{n}-\alpha_{n-1} \gamma f\left(T_{n-1} x_{n-1}\right)-\left(I-\alpha_{n-1} A\right) T_{n-1} x_{n-1}\right\| \\
= & \| \alpha_{n} \gamma f\left(T_{n} x_{n}\right)-\alpha_{n} \gamma f\left(T_{n-1} x_{n-1}\right)+\alpha_{n} \gamma f\left(T_{n-1} x_{n-1}\right) \\
& \quad-\alpha_{n-1} \gamma f\left(T_{n-1} x_{n-1}\right)+\left(I-\alpha_{n} A\right) T_{n} x_{n}-\left(I-\alpha_{n} A\right) T_{n-1} x_{n-1} \\
& \quad+\left(I-\alpha_{n} A\right) T_{n-1} x_{n-1}-\left(I-\alpha_{n-1} A\right) T_{n-1} x_{n-1} \| \\
\leq & \alpha_{n} \gamma \alpha\left\|T_{n} x_{n}-T_{n-1} x_{n}\right\|+\left|\alpha_{n}-\alpha_{n-1}\right|\left\|\gamma f\left(T_{n-1} x_{n-1}\right)-A T_{n-1} x_{n-1}\right\| \\
& +\left\|I-\alpha_{n} A\right\|\left\|T_{n} x_{n}-T_{n-1} x_{n-1}\right\| \\
\leq & \alpha_{n} \gamma \alpha\left\|T_{n} x_{n}-T_{n} x_{n-1}\right\|+\alpha_{n} \gamma \alpha\left\|T_{n} x_{n-1}-T_{n-1} x_{n}\right\|+\left|\alpha_{n}-\alpha_{n-1}\right| M \\
& +\varphi(1)(1-\alpha \bar{\gamma})\left\|T_{n} x_{n}-T_{n} x_{n-1}\right\|+\varphi(1)(1-\alpha \bar{\gamma})\left\|T_{n} x_{n-1}-T_{n-1} x_{n-1}\right\| \\
\leq & \left(1-\alpha_{n}(\varphi(1) \bar{\gamma}-\gamma \alpha)\right)\left\|x_{n}-x_{n-1}\right\| \\
& +\left(1-\alpha_{n}(\varphi(1) \bar{\gamma}-\gamma \alpha)\right)\left\|T_{n} x_{n-1}-T_{n-1} x_{n-1}\right\|+\left|\alpha_{n}-\alpha_{n-1}\right| M \\
\leq & \left(1-\alpha_{n}(\varphi(1) \bar{\gamma}-\gamma \alpha)\right)\left\|x_{n}-x_{n-1}\right\|+\left\|T_{n} x_{n-1}-T_{n-1} x_{n-1}\right\|+\left|\alpha_{n}-\alpha_{n-1}\right| M,
\end{aligned}
$$

for all $n \geq 1$, where $M$ is a constant satisfying $M \geq \sup _{n \geq 1}\left\|\gamma f\left(T_{n-1} x_{n-1}\right)-A T_{n-1} x_{n-1}\right\|$. Putting $\mu_{n}=\left\|T_{n} x_{n-1}-T_{n-1} x_{n-1}\right\|+\left|\alpha_{n}-\alpha_{n-1}\right| M$. From AKTT-condition and (C3), we have

$$
\sum_{n=1}^{\infty} \mu_{n} \leq \sum_{n=1}^{\infty} \sup _{x \in\left\{x_{n}\right\}}\left\|T_{n} x-T_{n-1} x\right\|+\sum_{n=1}^{\infty}\left|\alpha_{n}-\alpha_{n-1}\right| M<\infty
$$

Therefore, it follows from Lemma 2.2 that $\lim _{n \rightarrow \infty}\left\|x_{n+1}-x_{n}\right\|=0$. Since $\lim _{n \rightarrow \infty} \alpha_{n}=0$, we obtain

$$
\begin{aligned}
\left\|T_{n} x_{n}-x_{n}\right\| & \leq\left\|x_{n}-x_{n+1}\right\|+\left\|x_{n+1}-T_{n} x_{n}\right\| \\
& \leq\left\|x_{n}-x_{n+1}\right\|+\alpha_{n}\left\|\gamma f\left(T_{n} x_{n}\right)-A T_{n} x_{n}\right\| \longrightarrow 0 .
\end{aligned}
$$

Using Lemma 2.5, we obtain

$$
\begin{aligned}
\left\|T x_{n}-x_{n}\right\| & \leq\left\|T x_{n}-T_{n} x_{n}\right\|+\left\|T_{n} x_{n}-x_{n}\right\| \\
& \leq \sup \left\{\left\|T z-T_{n} z\right\|: z \in\left\{x_{n}\right\}\right\}+\left\|T_{n} x_{n}-x_{n}\right\| \longrightarrow 0 .
\end{aligned}
$$

Next, we prove that

$$
\limsup _{n \rightarrow \infty}\left\langle\gamma f(\tilde{x})-A \tilde{x}, J_{\varphi}\left(x_{n}-\tilde{x}\right)\right\rangle \leq 0
$$


Let $\left\{x_{n_{k}}\right\}$ be a subsequence of $\left\{x_{n}\right\}$ such that

$$
\lim _{k \rightarrow \infty}\left\langle\gamma f(\tilde{x})-A \tilde{x}, J_{\varphi}\left(x_{n_{k}}-\tilde{x}\right)\right\rangle=\limsup _{n \rightarrow \infty}\left\langle\gamma f(\tilde{x})-A \tilde{x}, J_{\varphi}\left(x_{n}-\tilde{x}\right)\right\rangle .
$$

If follows from reflexivity of $E$ and the boundedness of a sequence $\left\{x_{n_{k}}\right\}$ that there exists $\left\{x_{n_{k_{i}}}\right\}$ which is a subsequence of $\left\{x_{n_{k}}\right\}$ converging weakly to $w \in E$ as $i \rightarrow \infty$. Since $J_{\varphi}$ is weakly continuous, we have by Lemma 2.1 that

$$
\limsup _{n \rightarrow \infty} \Phi\left(\left\|x_{n_{k_{i}}}-x\right\|\right)=\limsup _{n \rightarrow \infty} \Phi\left(\left\|x_{n_{k_{i}}}-w\right\|\right)+\Phi(\|x-w\|), \quad \forall x \in E .
$$

Let

$$
H(x)=\limsup _{n \rightarrow \infty} \Phi\left(\left\|x_{n_{k_{i}}}-x\right\|\right), \quad \forall x \in E .
$$

It follows that

$$
H(x)=H(w)+\Phi(\|x-w\|), \quad \forall x \in E .
$$

Then, from $\lim _{n \rightarrow \infty}\left\|x_{n}-T x_{n}\right\|=0$, we have

$$
\begin{aligned}
H(T w) & =\underset{i \rightarrow \infty}{\limsup } \Phi\left(\left\|x_{n_{k_{i}}}-T w\right\|\right)=\underset{i \rightarrow \infty}{\limsup } \Phi\left(\left\|T x_{n_{k_{i}}}-T w\right\|\right) \\
& \leq \limsup _{i \rightarrow \infty} \Phi\left(\left\|x_{n_{k_{i}}}-w\right\|\right)=H(w) .
\end{aligned}
$$

On the other hand, however,

$$
H(T w)=H(w)+\Phi(\|T(w)-w\|) .
$$

It follows from (3.14) and (3.15) that

$$
\Phi(\|T(w)-w\|)=H(T w)-H(w) \leq 0 .
$$

Therefore, $T w=w$, and hence $w \in F(T)$. Since the duality map $J_{\varphi}$ is single valued and weakly continuous, we obtain, by (3.1), that

$$
\begin{aligned}
\limsup _{n \rightarrow \infty}\left\langle\gamma f(\tilde{x})-A \tilde{x}, J_{\varphi}\left(x_{n}-\tilde{x}\right)\right\rangle & =\lim _{k \rightarrow \infty}\left\langle\gamma f(\tilde{x})-A \tilde{x}, J_{\varphi}\left(x_{n_{k}}-\tilde{x}\right)\right\rangle \\
& =\lim _{i \rightarrow \infty}\left\langle\gamma f(\tilde{x})-A \tilde{x}, J_{\varphi}\left(x_{n_{k_{i}}}-\tilde{x}\right)\right\rangle \\
& =\left\langle(A-\gamma f) \tilde{x}, J_{\varphi}(\tilde{x}-w)\right\rangle \leq 0 .
\end{aligned}
$$


Fixed Point Theory and Applications

Next, we show that $x_{n} \rightarrow \tilde{x}$ as $n \rightarrow \infty$. In fact, since $\Phi(t)=\int_{0}^{t} \varphi(\tau) d \tau$, for all $t \geq 0$, and $\varphi:[0, \infty) \rightarrow[0, \infty)$ is a gauge function, then for $1 \geq k \geq 0, \varphi(k x) \leq \varphi(x)$ and

$$
\Phi(k t)=\int_{0}^{k t} \varphi(\tau) d \tau=k \int_{0}^{t} \varphi(k x) d x \leq k \int_{0}^{t} \varphi(x) d x=k \Phi(t) .
$$

Finally, we show that $x_{n} \rightarrow \tilde{x}$ as $n \rightarrow \infty$. Following Lemma 2.1, we have

$$
\begin{aligned}
\Phi\left(\left\|x_{n+1}-\tilde{x}\right\|\right)= & \Phi\left(\left\|\alpha_{n}\left(\gamma f\left(T_{n} x_{n}\right)-A \tilde{x}\right)+\left(I-\alpha_{n} A\right) T_{n} x_{n}-\left(I-\alpha_{n} A\right) \tilde{x}\right\|\right) \\
\leq & \Phi\left(\left\|\left(I-\alpha_{n} A\right) T_{n} x_{n}-\left(I-\alpha_{n} A\right) \tilde{x}\right\|\right)+\alpha_{n}\left\langle\gamma f\left(T_{n} x_{n}\right)-A \tilde{x}, J_{\varphi}\left(x_{n+1}-\tilde{x}\right)\right\rangle \\
\leq & \varphi(1)\left(1-\alpha_{n} \bar{\gamma}\right) \Phi\left(\left\|T_{n} x_{n}-\tilde{x}\right\|\right)+\alpha_{n}\left\langle\gamma f\left(T_{n} x_{n}\right)-A \tilde{x}, J_{\varphi}\left(x_{n+1}-\tilde{x}\right)\right\rangle \\
\leq & \left(1-\alpha_{n} \bar{\gamma}\right) \Phi\left(\left\|x_{n}-\tilde{x}\right\|\right)+\alpha_{n}\left\langle\gamma f\left(T_{n} x_{n}\right)-A \tilde{x}, J_{\varphi}\left(x_{n+1}-\tilde{x}\right)\right\rangle \\
= & \left(1-\alpha_{n} \bar{\gamma}\right) \Phi\left(\left\|x_{n}-\tilde{x}\right\|\right)+\alpha_{n}\left\langle\gamma f\left(T_{n} x_{n}\right)-\gamma f\left(T_{n} x_{n+1}\right), J_{\varphi}\left(x_{n+1}-\tilde{x}\right)\right\rangle \\
& +\alpha_{n}\left\langle\gamma f\left(T_{n} x_{n+1}\right)-\gamma f(\tilde{x}), J_{\varphi}\left(x_{n+1}-\tilde{x}\right)\right\rangle+\alpha_{n}\left\langle\gamma f(\tilde{x})-A \tilde{x}, J_{\varphi}\left(x_{n+1}-\tilde{x}\right)\right\rangle \\
\leq & \left(1-\alpha_{n} \bar{\gamma}\right) \Phi\left(\left\|x_{n}-\tilde{x}\right\|\right)+\alpha_{n} \gamma \alpha\left\|x_{n}-x_{n+1}\right\|\left\|J_{\varphi}\left(x_{n+1}-\tilde{x}\right)\right\| \\
& +\alpha_{n} \gamma \alpha\left\|x_{n+1}-\tilde{x}\right\|\left\|J_{\varphi}\left(x_{n+1}-\tilde{x}\right)\right\|+\alpha_{n}\left\langle\gamma f(\tilde{x})-A \tilde{x}, J_{\varphi}\left(x_{n+1}-\tilde{x}\right)\right\rangle \\
= & \left(1-\alpha_{n} \bar{\gamma}\right) \Phi\left(\left\|x_{n}-\tilde{x}\right\|\right)+\alpha_{n} \gamma \alpha\left\|x_{n}-x_{n+1}\right\|\left\|J_{\varphi}\left(x_{n+1}-\tilde{x}\right)\right\| \\
& +\alpha_{n} \gamma \alpha \Phi\left(\left\|x_{n+1}-\tilde{x}\right\|\right)+\alpha_{n}\left\langle\gamma f(\tilde{x})-A \tilde{x}, J_{\varphi}\left(x_{n+1}-\tilde{x}\right)\right\rangle .
\end{aligned}
$$

It then follows that

$$
\begin{aligned}
\Phi( & \left.\left\|x_{n+1}-\tilde{x}\right\|\right) \\
\leq & \frac{1-\alpha_{n} \bar{\gamma}}{1-\alpha_{n} \gamma \alpha} \Phi\left(\left\|x_{n}-\tilde{x}\right\|\right) \\
& +\alpha_{n}\left[\frac{\gamma \alpha}{1-\alpha_{n} \gamma \alpha}\left\|x_{n}-x_{n+1}\right\| M^{\prime}+\frac{1}{1-\alpha_{n} \gamma \alpha}\left\langle\gamma f(\tilde{x})-A \tilde{x}, J_{\varphi}\left(x_{n+1}-\tilde{x}\right)\right\rangle\right] \\
= & \left(1-\frac{\alpha_{n}(\bar{\gamma}+\gamma \alpha)}{1-\alpha_{n} \gamma \alpha}\right) \Phi\left(\left\|x_{n}-\tilde{x}\right\|\right)+\alpha_{n} \frac{\bar{\gamma}+\gamma \alpha}{1-\alpha_{n} \gamma \alpha} \\
& \times\left[\frac{1-\alpha_{n} \gamma \alpha}{\bar{\gamma}+\gamma \alpha}\left(\frac{\gamma \alpha}{1-\alpha_{n} \gamma \alpha}\left\|x_{n}-x_{n+1}\right\| M^{\prime}+\frac{1}{1-\alpha_{n} \gamma \alpha}\left\langle\gamma f(\tilde{x})-A \tilde{x}, J_{\varphi}\left(x_{n+1}-\tilde{x}\right)\right\rangle\right)\right],
\end{aligned}
$$

where $M^{\prime}=\sup _{n \geq 0}\left\|J_{\varphi}\left(x_{n+1}-\tilde{x}\right)\right\|$. Put

$$
\begin{gathered}
\gamma_{n}=\frac{\alpha_{n}(\bar{\gamma}+\gamma \alpha)}{1-\alpha_{n} \gamma \alpha}, \\
\delta_{n}=\frac{1-\alpha_{n} \gamma \alpha}{\bar{\gamma}+\gamma \alpha}\left(\frac{\gamma \alpha}{1-\alpha_{n} \gamma \alpha}\left\|x_{n}-x_{n+1}\right\| M^{\prime}+\frac{1}{1-\alpha_{n} \gamma \alpha}\left\langle\gamma f(\tilde{x})-A \tilde{x}, J_{\varphi}\left(x_{n+1}-\tilde{x}\right)\right\rangle\right) .
\end{gathered}
$$


It follows that from condition (C1), $\lim _{n \rightarrow \infty}\left\|x_{n+1}-x_{n}\right\|=0$ and (3.9) that

$$
\lim _{n \rightarrow \infty} \gamma_{n}=0, \quad \sum_{n=1}^{\infty} \gamma_{n}=\infty, \quad \limsup _{n \rightarrow \infty} \delta_{n} \leq 0
$$

Applying Lemma 2.2 to (3.20), we conclude that $\Phi\left(\left\|x_{n+1}-\tilde{x}\right\|\right) \rightarrow 0$ as $n \rightarrow \infty$; that is, $x_{n} \rightarrow \tilde{x}$ as $n \rightarrow \infty$. This completes the proof.

Setting $\gamma=1, A \equiv I$, where $I$ is the identity mapping and $f(x)=x$ for all $x \in E$ in Theorem 3.1, we have the following result.

Corollary 3.2. Let E be a reflexive Banach space which admits a weakly continuous duality mapping $J_{\varphi}$ with gauge $\varphi$. Suppose that $\left\{T_{n}: E \rightarrow E\right\}$ is a countable family of nonexpansive mappings satisfying $F:=\bigcap_{n=0}^{\infty} F\left(T_{n}\right) \neq \emptyset$. Assume that $\left\{x_{n}\right\}$ is defined by, for $x_{0}, x \in E$,

$$
x_{n+1}=\alpha_{n} x+\left(1-\alpha_{n}\right) T_{n} x_{n}, \quad n \geq 0,
$$

where $\left\{\alpha_{n}\right\}$ is a sequence in $[0,1]$ satisfying the following conditions:

(C1) $\lim _{n \rightarrow \infty} \alpha_{n}=0$,

(C2) $\sum_{n=0}^{\infty} \alpha_{n}=\infty$,

(C3) $\sum_{n=0}^{\infty}\left|\alpha_{n}-\alpha_{n+1}\right|<\infty$.

Suppose that $\left\{T_{n}\right\}$ satisfies the AKTT-condition. Let $T$ be a mapping of $E$ into itself defined by $T z=$ $\lim _{n \rightarrow \infty} T_{n} z$ for all $z \in E$, and suppose that $F(T)=\cap_{n=0}^{\infty} F\left(T_{n}\right)$, then $\left\{x_{n}\right\}$ converges strongly to $\tilde{x}$ of $F$ which solves the variational inequality

$$
\left\langle(I-f) \tilde{x}, J_{\varphi}(\tilde{x}-p)\right\rangle \leq 0, \quad \forall p \in F .
$$

Applying Theorem 3.1, we can obtain the following two strong convergence theorems for the iterative sequences $\left\{z_{n}\right\}$ and $\left\{y_{n}\right\}$ defined by (1.17).

Theorem 3.3. Let $E$ be a reflexive Banach space which admits a weakly continuous duality mapping $J_{\varphi}$ with gauge $\varphi$ such that $\varphi$ is invariant on $[0,1]$. Let $\left\{T_{n}: E \rightarrow E\right\}_{n=0}^{\infty}$ be a countable family of nonexpansive mappings satisfying $F:=\bigcap_{n=0}^{\infty} F\left(T_{n}\right) \neq \emptyset$. Let $f$ be an $\alpha$-contraction and $A$ a strongly positive bounded linear operator with coefficient $\bar{\gamma}>0$ and $0<\gamma<\bar{\gamma} \varphi(1) / \alpha$. Let the sequence $\left\{z_{n}\right\}$ be generated by (1.17), where $\left\{\alpha_{n}\right\}$ is a sequence in $[0,1]$ satisfying the following conditions:

(C1) $\lim _{n \rightarrow \infty} \alpha_{n}=0$,

(C2) $\sum_{n=0}^{\infty} \alpha_{n}=\infty$,

(C3) $\sum_{n=0}^{\infty}\left|\alpha_{n}-\alpha_{n+1}\right|<\infty$. 
Suppose that $\left\{T_{n}\right\}$ satisfies the AKTT-condition. Let $T$ be a mapping of $E$ into itself defined by $T z=$ $\lim _{n \rightarrow \infty} T_{n} z$ for all $z \in E$, and suppose that $F(T)=\bigcap_{n=0}^{\infty} F\left(T_{n}\right)$, then $\left\{z_{n}\right\}$ converges strongly to $\tilde{x}$ which solves the variational inequality (3.1).

Proof. Let $\left\{x_{n}\right\}$ be the sequence given by $x_{0}=z_{0}$ and

$$
x_{n+1}=\alpha_{n} \gamma f\left(T_{n} x_{n}\right)+\left(I-\alpha_{n} A\right) T_{n} x_{n}, \quad n \geq 0 .
$$

Form Theorem 3.1, $x_{n} \rightarrow \tilde{x}$. We claim that $z_{n} \rightarrow \tilde{x}$. Applying Lemma 2.6, we estimate

$$
\begin{aligned}
\left\|x_{n+1}-z_{n+1}\right\| & \leq \alpha_{n} \gamma\left\|f\left(z_{n}\right)-f\left(T_{n} x_{n}\right)\right\|+\left\|I-\alpha_{n} A\right\|\left\|T_{n} x_{n}-T_{n} z_{n}\right\| \\
& \leq \alpha_{n} \gamma \alpha\left\|z_{n}-T_{n} x_{n}\right\|+\varphi(1)\left(1-\alpha_{n} \bar{\gamma}\right)\left\|x_{n}-z_{n}\right\| \\
& \leq \alpha_{n} \gamma \alpha\left\|z_{n}-T_{n} \tilde{x}\right\|+\alpha_{n} \gamma \alpha\left\|T_{n} \tilde{x}-T_{n} x_{n}\right\|+\varphi(1)\left(1-\alpha_{n} \bar{\gamma}\right)\left\|x_{n}-z_{n}\right\| \\
& \leq \alpha_{n} \gamma \alpha\left\|z_{n}-\tilde{x}\right\|+\alpha_{n} \gamma \alpha\left\|T_{n} \tilde{x}-T_{n} x_{n}\right\|+\varphi(1)\left(1-\alpha_{n} \bar{\gamma}\right)\left\|x_{n}-z_{n}\right\| \\
& \leq \alpha_{n} \gamma \alpha\left\|z_{n}-x_{n}\right\|+\alpha_{n} \gamma \alpha\left\|x_{n}-\tilde{x}\right\|+\alpha_{n} \gamma \alpha\left\|\tilde{x}-x_{n}\right\|+\varphi(1)\left(1-\alpha_{n} \bar{\gamma}\right)\left\|x_{n}-z_{n}\right\| \\
& =\left(1-\alpha_{n}(\varphi(1) \bar{\gamma}-\gamma \alpha)\right)\left\|x_{n}-z_{n}\right\|+\alpha_{n}(\varphi(1) \bar{\gamma}-\gamma \alpha) \frac{2 \alpha \gamma}{\varphi(1) \bar{\gamma}-\gamma \alpha}\left\|\tilde{x}-x_{n}\right\| .
\end{aligned}
$$

It follows from $\sum_{n=1}^{\infty} \alpha_{n}=\infty, \lim _{n \rightarrow \infty}\left\|x_{n}-\tilde{x}\right\|=0$, and Lemma 2.2 that $\left\|x_{n}-z_{n}\right\| \rightarrow 0$. Consequently, $z_{n} \rightarrow \tilde{x}$ as required.

Theorem 3.4. Let $E$ be a reflexive Banach space which admits a weakly continuous duality mapping $J_{\varphi}$ with gauge $\varphi$ such that $\varphi$ is invariant on $[0,1]$. Let $\left\{T_{n}: E \rightarrow E\right\}_{n=0}^{\infty}$ be a countable family of nonexpansive mappings satisfying $F:=\bigcap_{n=0}^{\infty} F\left(T_{n}\right) \neq \emptyset$. Let $f$ be an $\alpha$-contraction and $A$ a strongly positive bounded linear operator with coefficient $\bar{\gamma}>0$ and $0<\gamma<\bar{\gamma} \varphi(1) / \alpha$. Let the sequence $\left\{y_{n}\right\}$ be generated by (1.17), where $\left\{\alpha_{n}\right\}$ is sequence in $[0,1]$ satisfying the following conditions:

(C1) $\lim _{n \rightarrow \infty} \alpha_{n}=0$,

(C2) $\sum_{n=0}^{\infty} \alpha_{n}=\infty$,

(C3) $\sum_{n=0}^{\infty}\left|\alpha_{n}-\alpha_{n+1}\right|<\infty$.

Suppose that $\left\{T_{n}\right\}$ satisfies the AKTT-condition. Let $T$ be a mapping of $E$ into itself defined by $T z=$ $\lim _{n \rightarrow \infty} T_{n} z$ for all $z \in E$, and suppose that $F(T)=\bigcap_{n=0}^{\infty} F\left(T_{n}\right)$, then $\left\{y_{n}\right\}$ converges strongly to $\tilde{x}$ which solves the variational inequality (3.1).

Proof. Let the sequences $\left\{z_{n}\right\}$ and $\left\{\beta_{n}\right\}$ be given by

$$
z_{n}=\alpha_{n} \gamma f\left(y_{n}\right)+\left(I-\alpha_{n} A\right) y_{n}, \quad \beta_{n}=\alpha_{n+1} \quad \forall n \in \mathbb{N}
$$


Taking $p \in \bigcap_{n=0}^{\infty} F\left(T_{n}\right)$, we have

$$
\begin{aligned}
\left\|y_{n+1}-p\right\| & =\left\|T_{n} z_{n}-T_{n} p\right\| \leq\left\|z_{n}-p\right\| \\
& =\left\|\alpha_{n} \gamma f\left(y_{n}\right)+\left(I-\alpha_{n} A\right) y_{n}-p\right\| \\
& \leq\left(1-\alpha_{n}(\varphi(1) \bar{\gamma}-\gamma \alpha)\right)\left\|y_{n}-p\right\|+\alpha_{n}\|\gamma f(p)-A(p)\| \\
& =\left(1-\alpha_{n}(\varphi(1) \bar{\gamma}-\gamma \alpha)\right)\left\|y_{n}-p\right\|+\alpha_{n}(\varphi(1) \bar{\gamma}-\gamma \alpha) \frac{\|\gamma f(p)-A(p)\|}{\varphi(1) \bar{\gamma}-\gamma \alpha} .
\end{aligned}
$$

It follows from induction that

$$
\left\|y_{n+1}-p\right\| \leq \max \left\{\left\|y_{0}-p\right\|, \frac{\|\gamma f(p)-A(p)\|}{\varphi(1) \bar{\gamma}-\gamma \alpha}\right\}, \quad n \geq 0
$$

Thus, both $\left\{y_{n}\right\}$ and $\left\{z_{n}\right\}$ are bounded. We observe that

$$
z_{n+1}=\alpha_{n+1} \gamma f\left(y_{n+1}\right)+\left(I-\alpha_{n+1} A\right) y_{n+1}=\beta_{n} \gamma f\left(T_{n} z_{n}\right)+\left(I-\beta_{n} A\right) T_{n} z_{n}
$$

Thus, Theorem 3.1 implies that $\left\{z_{n}\right\}$ converges strongly to some point $\tilde{x}$. In this case, we also have

$$
\left\|y_{n}-\tilde{x}\right\| \leq\left\|y_{n}-z_{n}\right\|+\left\|z_{n}-\tilde{x}\right\|=\alpha_{n}\left\|\gamma f\left(y_{n}\right)-A y_{n}\right\|+\left\|z_{n}-\tilde{x}\right\| \longrightarrow 0 .
$$

Hence, the sequence $\left\{y_{n}\right\}$ converges strongly to $\tilde{x}$. This competes the proof.

Setting $r=1, A \equiv I$, where $I$ is the identity mapping and $f(x)=x$ for all $x \in E$ in Theorem 3.4, we have the following result.

Corollary 3.5. Let E be a reflexive Banach space which admits a weakly continuous duality mapping $J_{\varphi}$ with gauge $\varphi$. Suppose that $\left\{T_{n}: E \rightarrow E\right\}$ is a countable family of nonexpansive mappings satisfying $F:=\bigcap_{n=0}^{\infty} F\left(T_{n}\right) \neq \emptyset$. Assume that $\left\{x_{n}\right\}$ is defined by for $x_{0}, x \in E$,

$$
x_{n+1}=T_{n}\left(\alpha_{n} x+\left(1-\alpha_{n}\right) x_{n}\right), \quad n \geq 0,
$$

where $\left\{\alpha_{n}\right\}$ is a sequence in $[0,1]$ satisfying the following conditions:

(C1) $\lim _{n \rightarrow \infty} \alpha_{n}=0$,

(C2) $\sum_{n=0}^{\infty} \alpha_{n}=\infty$,

(C3) $\sum_{n=0}^{\infty}\left|\alpha_{n}-\alpha_{n+1}\right|<\infty$. 
Suppose that $\left\{T_{n}\right\}$ satisfies the AKTT-condition. Let $T$ be a mapping of $E$ into itself defined by $T z=$ $\lim _{n \rightarrow \infty} T_{n} z$ for all $z \in E$, and suppose that $F(T)=\bigcap_{n=0}^{\infty} F\left(T_{n}\right)$, then $\left\{x_{n}\right\}$ converges strongly to $\tilde{x}$ of $F$ which solves the variational inequality

$$
\left\langle(I-f) \tilde{x}, J_{\varphi}(\tilde{x}-p)\right\rangle \leq 0, \quad \forall p \in F .
$$

\section{Applications}

\section{1. $W$-Mappings}

Let $T_{1}, T_{2}, \ldots$ be infinite mappings of $C$ into itself, and let $\left\{\xi_{i}\right\}$ be a nonnegative real sequence with $0 \leq \xi_{i}<1$, for all $i \geq 1$. For any $n \in \mathbb{N}$, define a mapping $W_{n}$ of $C$ into itself as follows:

$$
\begin{aligned}
U_{n, n+1} & =I, \\
U_{n, n} & =\xi_{n} T_{n} U_{n, n+1}+\left(1-\xi_{n}\right) I, \\
U_{n, n-1} & =\xi_{n-1} T_{n-1} U_{n, n}+\left(1-\xi_{n-1}\right) I, \\
& \vdots \\
U_{n, k}= & \xi_{k} T_{k} U_{n, k+1}+\left(1-\xi_{k}\right) I, \\
U_{n, k-1}= & \xi_{k-1} T_{k-1} U_{n, k}+\left(1-\xi_{k-1}\right) I, \\
& \vdots \\
U_{n, 2}= & \mu_{2} T_{2} U_{n, 3}+\left(1-\xi_{2}\right) I, \\
W_{n}= & U_{n, 1}=\xi_{1} T_{1} U_{n, 2}+\left(1-\xi_{1}\right) I .
\end{aligned}
$$

Nonexpansivity of each $T_{i}$ ensures the nonexpansivity of $W_{n}$. The mapping $W_{n}$ is called a $W$-mapping generated by $T_{1}, T_{2}, \ldots, T_{n}$ and $\xi_{1}, \xi_{2}, \ldots, \xi_{n}$.

Throughout this section, we will assume that $0<\xi_{n} \leq \xi<1$, for all $n \geq 1$. Concerning $W_{n}$ defined by (4.1), we have the following useful lemmas.

Lemma 4.1 (see [4]). Let $C$ be a nonempty closed convex subset of a a strictly convex, reflexive Banach space $E,\left\{T_{i}: C \rightarrow C\right\}$ a family of infinitely nonexpansive mapping with $\bigcap_{i=1}^{\infty} F\left(T_{i}\right) \neq \emptyset$, and $\left\{\xi_{i}\right\}$ a real sequence such that $0<\xi_{i} \leq \xi<1$, for all $i \geq 1$, then:

(1) $W_{n}$ is nonexpansive and $F\left(W_{n}\right)=\bigcap_{i=1}^{\infty} F\left(T_{i}\right)$ for each $n \geq 1$;

(2) for each $x \in C$ and for each positive integer $k$, the limit $\lim _{n \rightarrow \infty} U_{n, k} x$ exists;

(3) the mapping $W: C \rightarrow C$ define by

$$
W x:=\lim _{n \rightarrow \infty} W_{n} x=\lim _{n \rightarrow \infty} U_{n, 1} x, \quad x \in C
$$

is a nonexpansive mapping satisfying $F(W)=\bigcap_{i=1}^{\infty} F\left(T_{i}\right)$, and it is called the $W$-mapping generated by $T_{1}, T_{2}, \ldots$ and $\xi_{1}, \xi_{2}, \ldots$ 
From Remark 3.1 of Peng and Yao [29], we obtain the following lemma.

Lemma 4.2. Let $E$ be a strictly convex, reflexive Banach space, $\left\{T_{i}: E \rightarrow E\right\}$ a family of infinitely nonexpansive mappings with $\bigcap_{i=1}^{\infty} F\left(T_{i}\right) \neq \emptyset$, and $\left\{\xi_{i}\right\}$ a real sequence such that $0<\xi_{i} \leq \xi<1$, for all $i \geq 1$. Then sequence $\left\{W_{n}\right\}$ satisfies the AKTT-condition.

Applying Lemma 4.2 and Theorem 3.1, we obtain the following result.

Theorem 4.3. Let $E$ be a reflexive Banach space which admits a weakly continuous duality mapping $J_{\varphi}$ with gauge $\varphi$ such that $\varphi$ is invariant on $[0,1]$. Let $\left\{T_{n}: E \rightarrow E\right\}_{n=1}^{\infty}$ be a countable family of nonexpansive mappings with $F:=\bigcap_{n=1}^{\infty} F\left(T_{n}\right) \neq \emptyset$ and $f$ an $\alpha$-contraction and $A$ a strongly positive bounded linear operator with coefficient $\bar{\gamma}>0$ and $0<\gamma<\bar{\gamma} \varphi(1) / \alpha$. Let the sequence $\left\{x_{n}\right\}$ be generated by the following:

$$
x_{1}=x \in E, \quad x_{n+1}=\alpha_{n} \gamma f\left(W_{n} x_{n}\right)+\left(I-\alpha_{n} A\right) W_{n} x_{n},
$$

where $\left\{W_{n}\right\}$ is defined by (4.1) and $\left\{\alpha_{n}\right\}$ is a sequence in $[0,1]$ satisfying the conditions (C1), (C2), and (C3). Then $\left\{x_{n}\right\}$ converges strongly to $\tilde{x}$ in $F$.

Applying Lemma 4.2 and Theorem 3.3, we obtain the following result.

Theorem 4.4. Let $E,\left\{T_{n}\right\},\left\{W_{n}\right\}, f, A$, and $\left\{\alpha_{n}\right\}$ be as in Theorem 4.3. Let the sequence $\left\{z_{n}\right\}$ be generated by the following:

$$
z_{1}=z \in E, \quad z_{n+1}=\alpha_{n} \gamma f\left(z_{n}\right)+\left(I-\alpha_{n} A\right) W_{n} z_{n}
$$

then $\left\{z_{n}\right\}$ converges strongly to $\tilde{x}$ in $F$.

Applying Lemma 4.2 and Theorem 3.4, we obtain the following result.

Theorem 4.5. Let $E,\left\{T_{n}\right\},\left\{W_{n}\right\}, f, A$, and $\left\{\alpha_{n}\right\}$ be as in Theorem 4.3. Let the sequence $\left\{y_{n}\right\}$ be generated by the following:

$$
y_{1}=y \in E, \quad y_{n+1}=W_{n}\left(\alpha_{n} \gamma f\left(y_{n}\right)+\left(I-\alpha_{n} A\right) y_{n}\right),
$$

then $\left\{y_{n}\right\}$ converges strongly to $\tilde{x}$ in $F$.

\subsection{Accretive Operators}

We consider the problem of finding a zero of an accretive operator. An operator $\Psi \subset E \times E$ is said to be accretive if for each $\left(x_{1}, y_{1}\right)$ and $\left(x_{2}, y_{2}\right) \in \Psi$, there exists $j \in J\left(x_{1}-x_{2}\right)$ such that $\left\langle y_{1}-\right.$ $\left.y_{2}, j\right\rangle \geq 0$. An accretive operator $\Psi$ is said to satisfy the range condition if $\overline{D(\Psi)} \subset R(I+\lambda \Psi)$ for all $\lambda>0$, where $D(\Psi)$ is the domain of $\Psi, I$ is the identity mapping on $E, R(I+\lambda \Psi)$ is the range of $I+\lambda \Psi$, and $\overline{D(\Psi)}$ is the closure of $D(\Psi)$. If $\Psi$ is an accretive operator which satisfies the range condition, then we can define, for each $\lambda>0$, a mapping $J_{\lambda}: R(I+\lambda \Psi) \rightarrow D(\Psi)$ by $J_{\lambda}=(I-\lambda \Psi)^{-1}$, which is called the resolvent of $\Psi$. We know that $J_{\lambda}$ is nonexpansive 
and $F\left(J_{\lambda}\right)=\Psi^{-1}(0)$ for all $\lambda>0$. We also know the following [30]: for each $\lambda, \mu>0$ and $x \in R(I+\lambda \Psi) \cap R(I+\mu \Psi)$, it holds that

$$
\left\|J_{\lambda} x-J_{\mu} x\right\| \leq \frac{|\lambda-\mu|}{\lambda}\left\|x-J_{\lambda} x\right\|
$$

From the Resolvent identity, we have the following lemma.

Lemma 4.6. Let $E$ be a Banach space and $C$ a nonempty closed convex subset of $E$. Let $\Psi \subseteq E \times E$ be an accretive operator such that $\Psi^{-1} 0 \neq \emptyset$ and $\overline{D(\Psi)} \subset C \subset \bigcap_{\lambda>0} R(I+\lambda \Psi)$. Suppose that $\left\{\lambda_{n}\right\}$ is a sequence of $(0, \infty)$ such that $\inf \left\{\lambda_{n}: n \in \mathbb{N}\right\}>0$ and $\sum_{n=1}^{\infty}\left|\lambda_{n+1}-\lambda_{n}\right|<\infty$, then

(i) the sequence $\left\{J_{\lambda_{n}}\right\}$ satisfies AKTT-condition,

(ii) $\lim _{n \rightarrow \infty} J_{\lambda_{n}} z=J_{\lambda} z$ for all $z \in C$ and $F\left(J_{\curlywedge}\right)=\bigcap_{n=1}^{\infty} F\left(J_{\lambda_{n}}\right)$, where $\lambda_{n} \rightarrow \lambda$ as $n \rightarrow \infty$.

Proof. By the proof of Theorem 4.3 in [1] and applying Lemma 4.6 and Theorem 3.1, we obtain the following result.

Theorem 4.7. Let $E$ be a reflexive Banach space which admits a weakly continuous duality mapping $J_{\varphi}$ with gauge $\varphi$ such that $\varphi$ is invariant on $[0,1]$. Let $\Psi: D(\Psi) \subset E \rightarrow 2^{E}$ be an accretive operator such that $\Psi^{-1} 0 \neq \emptyset$. Assume that $K$ is a nonempty closed convex subset of $E$ such that $\overline{D(\Psi)} \subset$ $K \subset \bigcap_{\lambda>0} R(I+\lambda \Psi)$ and $f$ is an $\alpha$-contraction. Let $A$ be a strongly positive bounded linear operator with coefficient $\bar{\gamma}>0$ and $0<\gamma<\bar{\gamma} \varphi(1) / \alpha$. Suppose that $\left\{\lambda_{n}\right\}$ is a sequence of $(0, \infty)$ such that $\lim _{n \rightarrow \infty} \lambda_{n}=+\infty$. Let the sequence $\left\{x_{n}\right\}$ be generated by the following:

$$
x_{0}=x \in E, \quad x_{n+1}=\alpha_{n} \gamma f\left(J_{\lambda_{n}} x_{n}\right)+\left(I-\alpha_{n} A\right) J_{\lambda_{n}} x_{n}
$$

where $\left\{\alpha_{n}\right\}$ is a sequence in $[0,1]$ satisfying the following conditions (C1), (C2), and (C3), then $\left\{x_{n}\right\}$ converges strongly to $\tilde{x}$ in $\Psi^{-1} 0$.

Applying Lemma 4.6 and Theorem 3.3, we obtain the following result.

Theorem 4.8. Let $E, \Psi, K, f, A,\left\{\alpha_{n}\right\}$, and $\left\{\lambda_{n}\right\}$ be as in Theorem 4.7. Let $\left\{z_{n}\right\}$ be generated by the following:

$$
z_{0}=z \in E, \quad z_{n+1}=\alpha_{n} \gamma f\left(z_{n}\right)+\left(I-\alpha_{n} A\right) J_{\lambda_{n}} z_{n},
$$

then $\left\{z_{n}\right\}$ converges strongly to $\tilde{x}$ in $\Psi^{-1} 0$.

Applying Lemma 4.6 and Theorem 3.4, we obtain the following result.

Theorem 4.9. Let $E, \Psi, K, f, A,\left\{\alpha_{n}\right\}$, and $\left\{\lambda_{n}\right\}$ be as in Theorem 4.7. Let $\left\{y_{n}\right\}$ be generated by the following:

$$
y_{0}=z \in E, \quad y_{n+1}=J_{\lambda_{n}}\left(\alpha_{n} \gamma f\left(y_{n}\right)+\left(I-\alpha_{n} A\right) y_{n}\right),
$$

Then $\left\{y_{n}\right\}$ converges strongly to $\tilde{x}$ in $\Psi^{-1} 0$. 


\subsection{The Equilibrium Problems}

Let $H$ be a real Hilbert space, and let $F$ be a bifunction of $H \times H \rightarrow \mathbb{R}$, where $\mathbb{R}$ is the set of real numbers. The equilibrium problem for $F: H \times H \rightarrow \mathbb{R}$ is to find $x \in H$ such that

$$
F(x, y) \geq 0, \quad \forall y \in H
$$

The set of solutions of (4.10) is denoted by $\mathrm{EP}(F)$. Given a mapping $T: H \rightarrow H$, let $F(x, y)=$ $\langle T x, y-x\rangle$ for all $x, y \in H$. Then, $z \in \operatorname{EP}(F)$ if and only if $\langle T x, y-z\rangle \geq 0$ for all $y \in H$, that is, $z$ is a solution of the variational inequality. Numerous problems in physics, optimization, and economics reduce to find a solution of (4.10). Some methods have been proposed to solve the equilibrium problem; see, for instance, Blum and Oettli [31] and Combettes and Hirstoaga [32]. For the purpose of solving the equilibrium problem for a bifunction $F$, let us assume that $F$ satisfies the following conditions:

(A1) $F\langle x, x\rangle=0$ for all $x \in H$,

(A2) $F$ is monotone, that is, $F(x, y)+F(y, x) \leq 0$ for all $x, y \in H$,

(A3) for each $x, y, z \in H, \lim _{t \rightarrow 0} F(t z+(1-t) x, y) \leq F(x, y)$,

(A4) for each $x \in H, y \mapsto F(x, y)$ is convex and lower semicontinuous.

The following lemmas were also given in $[31,32]$, respectively.

Lemma 4.10 (see [31, Corollary 1]). Let $C$ be a nonempty closed convex subset of $H$, and let $F$ be a bifunction of $C \times C \rightarrow \mathbb{R}$ satisfying $(A 1)-(A 4)$. Let $r>0$ and $x \in H$, then there exists $z \in C$ such that $F(z, y)+(1 / r)\langle y-z, z-x\rangle \geq 0$ for all $y \in C$.

Lemma 4.11 (see [32, Lemma 2.12]). Assume that $F: C \times C \in \mathbb{R}$ satisfies (A1)-(A4). For $r>0$ and $x \in H$, define a mapping $T_{r}: H \rightarrow C$ as follows:

$$
T_{r}(x)=\left\{z \in C ; F(z, y)+\frac{1}{r}\langle y-z, z-x\rangle \geq 0, \forall y \in C\right\} \quad \forall x \in H,
$$

then, the following hold:

(1) $T_{r}$ is single valued,

(2) $T_{r}$ is firmly nonexpansive, that is, for any $x, y \in H,\left\|T_{r} x-T_{r} y\right\|^{2} \leq\left\langle T_{r} x-T_{r} y, x-y\right\rangle$,

(3) $F\left(T_{r}\right)=E P(F)$,

(4) $E P(F)$ is closed and convex.

Theorem 4.12. Let $H$ be a real Hilbert space. Let $F$ be a bifunction from $H \times H \rightarrow \mathbb{R}$ satisfying (A1)-(A4) and $E P(F) \neq \emptyset$. Let $f$ be an $\alpha$-contraction, $A$ a strongly positive bounded linear operator with coefficient $\bar{\gamma}>0$ and $0<\gamma<\bar{\gamma} / \alpha$. Let the sequences $\left\{x_{n}\right\},\left\{u_{n}\right\}$ be generated by $x_{0} \in H$ and

$$
\begin{gathered}
F\left(u_{n}, y\right)+\frac{1}{r_{n}}\left\langle y-u_{n}, u_{n}-x_{n}\right\rangle \geq 0, \quad \forall y \in H, \\
x_{n+1}=\alpha_{n} \gamma f\left(u_{n}\right)+\left(I-\alpha_{n} A\right) u_{n}
\end{gathered}
$$


for all $n \geq 0$, where $\left\{\alpha_{n}\right\}$ is a sequence in $[0,1]$ and $r_{n} \in(0, \infty)$ satisfying the following conditions:

(C1) $\lim _{n \rightarrow \infty} \alpha_{n}=0$,

(C2) $\sum_{n=1}^{\infty} \alpha_{n}=\infty$,

(C3) $\sum_{n=1}^{\infty}\left|\alpha_{n+1}-\alpha_{n}\right|<\infty$,

(C4) $\liminf _{n \rightarrow \infty} r_{n}>0$ and $\sum_{n=1}^{\infty}\left|r_{n+1}-r_{n}\right|<\infty$.

then $\left\{x_{n}\right\}$ and $\left\{u_{n}\right\}$ converge strongly to $\tilde{x} \in E P(F)$.

Proof. Following the proof technique of Theorem 3.1, we only need, show that $\lim _{n \rightarrow \infty} \| x_{n}-$ $T_{r} x_{n} \|=0$, for all $r>0$. From (4.12), it follows that

$$
\begin{aligned}
\left\|x_{n+2}-x_{n+1}\right\|= & \left\|\alpha_{n+1} \gamma f\left(u_{n+1}\right)+\left(I-\alpha_{n+1} A\right) u_{n+1}-\alpha_{n} \gamma f\left(u_{n}\right)-\left(I-\alpha_{n} A\right) u_{n}\right\| \\
= & \| \alpha_{n+1} \gamma f\left(u_{n+1}\right)+\left(I-\alpha_{n+1} A\right) u_{n+1}-\left(I-\alpha_{n+1} A\right) u_{n}+\left(I-\alpha_{n+1} A\right) u_{n} \\
& -\alpha_{n} \gamma f\left(u_{n}\right)-\left(I-\alpha_{n} A\right) u_{n}-\alpha_{n+1} \gamma f\left(u_{n}\right)+\alpha_{n+1} \gamma f\left(u_{n}\right) \| \\
\leq & \left\|\left(I-\alpha_{n+1} A\right)\left(u_{n+1}-u_{n}\right)\right\|+\alpha_{n+1} \gamma\left\|f\left(u_{n+1}\right)-f\left(u_{n}\right)\right\| \\
& +\left|\alpha_{n+1}-\alpha_{n}\right| \gamma\left\|f\left(u_{n}\right)\right\|+\left\|\left(\alpha_{n} A-\alpha_{n+1} A\right) u_{n}\right\| \\
= & \left\|\left(I-\alpha_{n+1} A\right)\left(u_{n+1}-u_{n}\right)\right\|+\alpha_{n+1} \gamma\left\|f\left(u_{n+1}\right)-f\left(u_{n}\right)\right\| \\
& +\left|\alpha_{n+1}-\alpha_{n}\right| \gamma\left\|f\left(u_{n}\right)\right\|+\left\|\left(\alpha_{n}-\alpha_{n+1}\right) A u_{n}\right\| \\
\leq & \left(1-\alpha_{n+1} \bar{\gamma}\right)\left\|T_{r_{n+1}} x_{n+1}-T_{r_{n}} x_{n}\right\|+\alpha_{n+1} \gamma\left\|f\left(u_{n+1}\right)-f\left(u_{n}\right)\right\| \\
& +\left|\alpha_{n+1}-\alpha_{n}\right| \gamma\left\|f\left(u_{n}\right)\right\|+\left|\alpha_{n}-\alpha_{n+1}\right|\left\|A u_{n}\right\| \\
= & \left(1-\alpha_{n+1} \bar{\gamma}\right)\left\|T_{r_{n+1}} x_{n+1}-T_{r_{n+1}} x_{n}+T_{r_{n+1}} x_{n}-T_{r_{n}} x_{n}\right\| \\
& +\alpha_{n+1} \gamma\left\|f\left(u_{n+1}\right)-f\left(u_{n}\right)\right\|+\left|\alpha_{n+1}-\alpha_{n}\right| \gamma\left\|f\left(u_{n}\right)\right\|+\left|\alpha_{n}-\alpha_{n+1}\right|\left\|A u_{n}\right\| \\
\leq & \left(1-\alpha_{n+1} \bar{\gamma}\right)\left\|T_{r_{n+1}} x_{n+1}-T_{r_{n+1}} x_{n}\right\|+\left(1-\alpha_{n+1} \bar{\gamma}\right)\left\|T_{r_{n+1}} x_{n}-T_{r_{n}} x_{n}\right\| \\
& +\alpha_{n+1} \gamma\left\|f\left(u_{n+1}\right)-f\left(u_{n}\right)\right\|+\left|\alpha_{n+1}-\alpha_{n}\right| \gamma\left\|f\left(u_{n}\right)\right\|+\left|\alpha_{n}-\alpha_{n+1}\right|\left\|A u_{n}\right\| \\
\leq & \left(1-\alpha_{n+1} \bar{\gamma}\right)\left\|x_{n+1}-x_{n}\right\|+\left(1-\alpha_{n+1} \bar{\gamma}\right)\left\|T_{r_{n+1}} x_{n}-T_{r_{n}} x_{n}\right\| \\
& +\alpha_{n+1} \gamma \alpha\left\|u_{n+1}-u_{n}\right\|+\left|\alpha_{n+1}-\alpha_{n}\right| \gamma\left\|f\left(u_{n}\right)\right\|+\left|\alpha_{n}-\alpha_{n+1}\right|\left\|A u_{n}\right\| \\
\leq & \left(1-\alpha_{n+1} \bar{\gamma}\right)\left\|x_{n+1}-x_{n}\right\|+\left(1-\alpha_{n+1} \bar{\gamma}\right)\left\|T_{r_{n+1}} x_{n}-T_{r_{n}} x_{n}\right\|+\alpha_{n+1} \gamma \alpha\left\|x_{n+1}-x_{n}\right\| \\
& +\alpha_{n+1} \gamma \alpha\left\|T_{r_{n+1}} x_{n}-T_{r_{n}} x_{n}\right\|+\left|\alpha_{n+1}-\alpha_{n}\right| \gamma\left\|f\left(u_{n}\right)\right\|+\left|\alpha_{n}-\alpha_{n+1}\right|\left\|A u_{n}\right\| \\
= & \left(1-\alpha_{n+1}(\bar{\gamma}-\gamma \alpha)\right)\left\|x_{n+1}-x_{n}\right\|+\left(1-\alpha_{n+1}(\bar{\gamma}-\gamma \alpha)\right)\left\|T_{r_{n+1}} x_{n}-T_{r_{n}} x_{n}\right\| \\
& +\left|\alpha_{n+1}-\alpha_{n}\right| \gamma\left\|f\left(u_{n}\right)\right\|+\left|\alpha_{n}-\alpha_{n+1}\right|\left\|A u_{n}\right\| . \\
&
\end{aligned}
$$


On the other hand, from the definition of $T_{r}$ we have

$$
\begin{gathered}
F\left(T_{r_{n}} x_{n}, y\right)+\frac{1}{r_{n}}\left\langle y-T_{r_{n}} x_{n}, T_{r_{n}} x_{n}-x_{n}\right\rangle \geq 0, \quad \forall y \in H, \\
F\left(T_{r_{n+1}} x_{n}, y\right)+\frac{1}{r_{n}}\left\langle y-T_{r_{n+1}} x_{n}, T_{r_{n+1}} x_{n}-x_{n}\right\rangle \geq 0, \quad \forall y \in H .
\end{gathered}
$$

Putting $y=T_{r_{n+1}} x_{n}$ and $y=T_{r_{n}} x_{n}$ in (4.14), we have

$$
\begin{gathered}
F\left(T_{r_{n}} x_{n}, T_{r_{n+1}} x_{n}\right)+\frac{1}{r_{n}}\left\langle T_{r_{n+1}} x_{n}-T_{r_{n}} x_{n}, y, T_{r_{n}} x_{n}, T_{r_{n+1}} x_{n}-x_{n}\right\rangle \geq 0, \\
F\left(T_{r_{n+1}} x_{n}, T_{r_{n}} x_{n}\right)+\frac{1}{r_{n}}\left\langle T_{r_{n}} x_{n}-T_{r_{n+1}} x_{n}, T_{r_{n}} x_{n}, T_{r_{n+1}} x_{n}, T_{r_{n}} x_{n}-x_{n}\right\rangle \geq 0 .
\end{gathered}
$$

So, from (A2), we have

$$
\left\langle T_{r_{n}} x_{n}-T_{r_{n+1}} x_{n}, \frac{T_{r_{n+1}} x_{n}-x_{n}}{r_{n+1}}-\frac{T_{r_{n}} x_{n}-x_{n}}{r_{n}}\right\rangle \geq 0,
$$

and hence,

$$
\left\langle T_{r_{n}} x_{n}-T_{r_{n+1}} x_{n}, \frac{T_{r_{n+1}} x_{n}-T_{r_{n}} x_{n}}{r_{n+1}}+\left(\frac{1}{r_{n+1}}-\frac{1}{r_{n}}\right)\left(T_{r_{n}} x_{n}-x_{n}\right)\right\rangle \geq 0,
$$

then we have

$$
\begin{aligned}
\frac{\left\|T_{r_{n+1}} x_{n}-T_{r_{n}} x_{n}\right\|^{2}}{r_{n+1}} & \leq\left\langle T_{r_{n}} x_{n}-T_{r_{n+1}} x_{n},\left(\frac{1}{r_{n+1}}-\frac{1}{r_{n}}\right)\left(T_{r_{n}} x_{n}-x_{n}\right)\right\rangle \\
& \leq\left\|T_{r_{n}} x_{n}-T_{r_{n+1}} x_{n}\right\|\left|\frac{1}{r_{n+1}}-\frac{1}{r_{n}}\right|\left\|T_{r_{n}} x_{n}-x_{n}\right\| \\
& \leq\left\|T_{r_{n}} x_{n}-T_{r_{n+1}} x_{n}\right\|\left|\frac{1}{r_{n+1}}-\frac{1}{r_{n}}\right| 2 M,
\end{aligned}
$$

and hence,

$$
\left\|T_{r_{n+1}} x_{n}-T_{r_{n}} x_{n}\right\| \leq\left|1-\frac{r_{n+1}}{r_{n}}\right| 2 M
$$

where $M$ is a constant satisfying $M \geq \sup _{n \geq 0}\left\|T_{r_{n}} x_{n}-x_{n}\right\|$. Substituting (4.19) in (4.13) yields

$$
\begin{aligned}
\left\|x_{n+2}-x_{n+1}\right\| \leq & \left(1-\alpha_{n+1}(\bar{\gamma}-\gamma \alpha)\right)\left\|x_{n+1}-x_{n}\right\|+\left(1-\alpha_{n+1}(\bar{\gamma}-\gamma \alpha)\right) \frac{2 M}{b}\left|r_{n+1}-r_{n}\right| \\
& +\left|\alpha_{n+1}-\alpha_{n}\right| \gamma\left\|f\left(u_{n}\right)\right\|+\left|\alpha_{n}-\alpha_{n+1}\right|\left\|A u_{n}\right\| \\
\leq & \left(1-\alpha_{n+1}(\bar{\gamma}-\gamma \alpha)\right)\left\|x_{n+1}-x_{n}\right\|+2 M\left|\alpha_{n+1}-\alpha_{n}\right|+\frac{2 M}{b}\left|r_{n+1}-r_{n}\right|
\end{aligned}
$$


for some $b$ with $r_{n}>b>0$ (the definition $\lim \inf _{n \rightarrow \infty} r_{n}>0$ ). By the assumptions on $\left\{r_{n}\right\}$ and $\left\{\alpha_{n}\right\}$ and using Lemma 2.2, we conclude that

$$
\lim _{n \rightarrow \infty}\left\|x_{n+1}-x_{n}\right\|=0
$$

From the definition of $x_{n}$ and $\lim _{n \rightarrow \infty} \alpha_{n}=0$, it follows that

$$
\begin{aligned}
\left\|x_{n+1}-u_{n}\right\| & =\left\|\alpha_{n} \gamma f\left(u_{n}\right)+\left(I-\alpha_{n} A\right) u_{n}-u_{n}\right\| \\
& =\left\|\alpha_{n} \gamma f\left(u_{n}\right)-\alpha_{n} A u_{n}\right\| \\
& =\alpha_{n}\left\|\gamma f\left(u_{n}\right)-A u_{n}\right\| \longrightarrow 0 .
\end{aligned}
$$

Combining (4.21) and (4.22), we have

$$
\lim _{n \rightarrow \infty}\left\|x_{n}-u_{n}\right\|=\lim _{n \rightarrow \infty}\left\|x_{n}-T_{r_{n}} x_{n}\right\|=0 .
$$

From the definition of $T_{r}$, it follows that

$$
F\left(T_{r} T_{r_{n}} x_{n}, y\right)+\frac{1}{r}\left\langle y-T_{r} T_{r_{n}} x_{n}, T_{r} T_{r_{n}} x_{n}-T_{r_{n}} x_{n}\right\rangle \geq 0, \quad \forall y \in H
$$

Putting $y=T_{r} T_{r_{n}} x_{n}$ in (4.14) and $y=T_{r_{n}} x_{n}$ in (4.24), we have

$$
\begin{gathered}
F\left(T_{r_{n}} x_{n}, T_{r} T_{r_{n}} x_{n}\right)+\frac{1}{r_{n}}\left\langle T_{r} T_{r_{n}} x_{n}-T_{r_{n}} x_{n}, T_{r_{n}} x_{n}-x_{n}\right\rangle \geq 0, \quad \forall y \in H, \\
F\left(T_{r} T_{r_{n}} x_{n}, T_{r_{n}} x_{n}\right)+\frac{1}{r}\left\langle T_{r_{n}} x_{n}-T_{r} T_{r_{n}} x_{n}, T_{r} T_{r_{n}} x_{n}-T_{r_{n}} x_{n}\right\rangle \geq 0, \quad \forall y \in H .
\end{gathered}
$$

So, from (A2), we have

$$
\left\langle T_{r_{n}} x_{n}-T_{r} T_{r_{n}} x_{n}, \frac{T_{r} T_{r_{n}} x_{n}-x_{n}}{r}-\frac{T_{r_{n}} x_{n}-x_{n}}{r_{n}}\right\rangle \geq 0,
$$

and hence, for each $r>0$,

$$
\begin{aligned}
\frac{\left\|T_{r} T_{r_{n}} x_{n}-T_{r_{n}} x_{n}\right\|^{2}}{r} & \leq\left\langle T_{r_{n}} x_{n}-T_{r} T_{r_{n}} x_{n}, \frac{1}{r_{n}}\left(x_{n}-T_{r_{n}} x_{n}\right)\right\rangle \\
& \leq\left\|T_{r} T_{r_{n}} x_{n}-T_{r_{n}} x_{n}\right\| \frac{1}{r_{n}}\left\|T_{r_{n}} x_{n}-x_{n}\right\|,
\end{aligned}
$$

then

$$
\left\|T_{r} T_{r_{n}} x_{n}-T_{r_{n}} x_{n}\right\| \leq \frac{r\left\|T_{r_{n}} x_{n}-x_{n}\right\|}{b}
$$


Since

$$
\begin{aligned}
\left\|x_{n}-T_{r} x_{n}\right\| & \leq\left\|x_{n}-T_{r_{n}} x_{n}\right\|+\left\|T_{r_{n}} x_{n}-T_{r} T_{r_{n}} x_{n}\right\|+\left\|T_{r} T_{r_{n}} x_{n}-T_{r} x_{n}\right\| \\
& \leq 2\left\|x_{n}-T_{r_{n}} x_{n}\right\|+\frac{r\left\|T_{r_{n}} x_{n}-x_{n}\right\|}{b}=\left(2+\frac{r}{b}\right)\left\|x_{n}-T_{r_{n}} x_{n}\right\|,
\end{aligned}
$$

then for each $r>0$, we have from (4.23)

$$
\lim _{n \rightarrow \infty}\left\|x_{n}-T_{r} x_{n}\right\|=0
$$

This completes the proof.

Applying Theorem 4.12, we can obtain the following result.

Corollary 4.13. Let $H$ be a real Hilbert space. Let $F$ be a bifunction from $H \times H \rightarrow \mathbb{R}$ satisfying $(A 1)-(A 4)$ and $\operatorname{EP}(F) \neq \emptyset$. Let $f$ be an $\alpha$-contraction, $A$ a strongly positive bounded linear operator with coefficient $\bar{\gamma}>0$ and $0<\gamma<\bar{\gamma} / \alpha$. Let the sequences $\left\{z_{n}\right\},\left\{u_{n}\right\}$ be generated by $z_{0} \in H$ and

$$
\begin{gathered}
F\left(u_{n}, y\right)+\frac{1}{r_{n}}\left\langle y-u_{n}, u_{n}-z_{n}\right\rangle \geq 0, \quad \forall y \in H \\
z_{n+1}=\alpha_{n} \gamma f\left(z_{n}\right)+\left(I-\alpha_{n} A\right) u_{n}
\end{gathered}
$$

for all $n \in N$, where $\left\{\alpha_{n}\right\}$ is a sequence in $[0,1]$ and $r_{n} \in(0, \infty)$ satisfying the following conditions:

(C1) $\lim _{n \rightarrow \infty} \alpha_{n}=0$,

(C2) $\sum_{n=1}^{\infty} \alpha_{n}=\infty$,

(C3) $\sum_{n=1}^{\infty}\left|\alpha_{n+1}-\alpha_{n}\right|<\infty$,

(C4) $\liminf _{n \rightarrow \infty} r_{n}>0$ and $\sum_{n=1}^{\infty}\left|r_{n+1}-r_{n}\right|<\infty$,

then $\left\{z_{n}\right\}$ and $\left\{u_{n}\right\}$ converge strongly to $\tilde{x} \in E P(F)$.

Proof. We observe that $u_{n}=T_{r_{n}} z_{n}$ for all $n \geq 0$. Then we rewrite the iterative sequence (4.31) by the following:

$$
z_{0} \in H, \quad z_{n+1}=\alpha_{n} \gamma f\left(z_{n}\right)+\left(I-\alpha_{n} A\right) T_{r_{n}} z_{n}
$$

Let $\left\{x_{n}\right\}$ be the sequence given by $x_{0}=z_{0}$ and

$$
x_{n+1}=\alpha_{n} \gamma f\left(T_{r_{n}} x_{n}\right)+\left(I-\alpha_{n} A\right) T_{r_{n}} x_{n} .
$$


Form Theorem 4.12, $x_{n} \rightarrow \tilde{x}$ in $\mathrm{EP}(F)$. We claim that $z_{n} \rightarrow \tilde{x}$. Applying Lemma 2.6, we estimate

$$
\begin{aligned}
\left\|x_{n+1}-z_{n+1}\right\| & \leq \alpha_{n} \gamma\left\|f\left(z_{n}\right)-f\left(T_{r_{n}} x_{n}\right)\right\|+\left\|I-\alpha_{n} A\right\|\left\|T_{r_{n}} x_{n}-T_{r_{n}} z_{n}\right\| \\
& \leq \alpha_{n} \gamma \alpha\left\|z_{n}-T_{r_{n}} x_{n}\right\|+\left(1-\alpha_{n} \bar{\gamma}\right)\left\|x_{n}-z_{n}\right\| \\
& \leq \alpha_{n} \gamma \alpha\left\|z_{n}-T_{r_{n}} \tilde{x}\right\|+\alpha_{n} \gamma \alpha\left\|T_{r_{n}} \tilde{x}-T_{r_{n}} x_{n}\right\|+\left(1-\alpha_{n} \bar{\gamma}\right)\left\|x_{n}-z_{n}\right\| \\
& \leq \alpha_{n} \gamma \alpha\left\|z_{n}-\tilde{x}\right\|+\alpha_{n} \gamma \alpha\left\|\tilde{x}-x_{n}\right\|+\left(1-\alpha_{n} \bar{\gamma}\right)\left\|x_{n}-z_{n}\right\| \\
& \leq \alpha_{n} \gamma \alpha\left\|z_{n}-x_{n}\right\|+\alpha_{n} \gamma \alpha\left\|x_{n}-\tilde{x}\right\|+\alpha_{n} \gamma \alpha\left\|\tilde{x}-x_{n}\right\|+\left(1-\alpha_{n} \bar{\gamma}\right)\left\|x_{n}-z_{n}\right\| \\
& =\left(1-\alpha_{n}(\bar{\gamma}-\gamma \alpha)\right)\left\|x_{n}-z_{n}\right\|+\alpha_{n}(\bar{\gamma}-\gamma \alpha) \frac{2 \alpha \gamma}{\bar{\gamma}-\gamma \alpha}\left\|\tilde{x}-x_{n}\right\| .
\end{aligned}
$$

It follows from $\sum_{n=1}^{\infty} \alpha_{n}=\infty, \lim _{n \rightarrow \infty}\left\|x_{n}-\tilde{x}\right\|=0$, and Lemma 2.2 that $\left\|x_{n}-z_{n}\right\| \rightarrow 0$ as $n \rightarrow \infty$. Consequently, $z_{n} \rightarrow \tilde{x}$ as required.

\section{Acknowledgments}

The authors would like to thank the Centre of Excellence in Mathematics, Thailand for financial support. Finally, They would like to thank the referees for reading this paper carefully, providing valuable suggestions and comments, and pointing out a major error in the original version of this paper.

\section{References}

[1] K. Aoyama, Y. Kimura, W. Takahashi, and M. Toyoda, "Approximation of common fixed points of a countable family of nonexpansive mappings in a Banach space," Nonlinear Analysis: Theory, Methods $\mathcal{E}$ Applications, vol. 67, no. 8, pp. 2350-2360, 2007.

[2] H. H. Bauschke, "The approximation of fixed points of compositions of nonexpansive mappings in Hilbert space," Journal of Mathematical Analysis and Applications, vol. 202, no. 1, pp. 150-159, 1996.

[3] M. Shang, Y. Su, and X. Qin, "Strong convergence theorems for a finite family of nonexpansive mappings," Fixed Point Theory and Applications, vol. 2007, Article ID 76971, 9 pages, 2007.

[4] K. Shimoji and W. Takahashi, "Strong convergence to common fixed points of infinite nonexpansive mappings and applications," Taiwanese Journal of Mathematics, vol. 5, no. 2, pp. 387-404, 2001.

[5] H. H. Bauschke and J. M. Borwein, "On projection algorithms for solving convex feasibility problems," SIAM Review, vol. 38, no. 3, pp. 367-426, 1996.

[6] P. L. Combettes, "Foundations of set theoretic estimation," Proceedings of the IEEE, vol. 81, no. 2, pp. 182-208, 1993.

[7] D. C. Youla, "Mathematical theory of image restoration by the method of convex projections," in Image Recovery: Theory and Applications, H. Stark, Ed., pp. 29-77, Academic Press, Orlando, Fla, USA, 1987.

[8] A. N. Iusem and A. R. De Pierro, “On the convergence of Han's method for convex programming with quadratic objective," Mathematical Programming. Series B, vol. 52, no. 2, pp. 265-284, 1991.

[9] F. E. Browder, "Fixed-point theorems for noncompact mappings in Hilbert space," Proceedings of the National Academy of Sciences of the United States of America, vol. 53, pp. 1272-1276, 1965.

[10] S. Reich, "Strong convergence theorems for resolvents of accretive operators in Banach spaces," Journal of Mathematical Analysis and Applications, vol. 75, no. 1, pp. 287-292, 1980.

[11] H.-K. Xu, "Strong convergence of an iterative method for nonexpansive and accretive operators," Journal of Mathematical Analysis and Applications, vol. 314, no. 2, pp. 631-643, 2006. 
[12] F. Deutsch and I. Yamada, "Minimizing certain convex functions over the intersection of the fixed point sets of nonexpansive mappings," Numerical Functional Analysis and Optimization, vol. 19, no. 1-2, pp. 33-56, 1998.

[13] H.-K. Xu, "An iterative approach to quadratic optimization," Journal of Optimization Theory and Applications, vol. 116, no. 3, pp. 659-678, 2003.

[14] H.-K. Xu, "Iterative algorithms for nonlinear operators," Journal of the London Mathematical Society. Second Series, vol. 66, no. 1, pp. 240-256, 2002.

[15] A. Moudafi, "Viscosity approximation methods for fixed-points problems," Journal of Mathematical Analysis and Applications, vol. 241, no. 1, pp. 46-55, 2000.

[16] H.-K. Xu, "Viscosity approximation methods for nonexpansive mappings," Journal of Mathematical Analysis and Applications, vol. 298, no. 1, pp. 279-291, 2004.

[17] G. Marino and H.-K. Xu, "A general iterative method for nonexpansive mappings in Hilbert spaces," Journal of Mathematical Analysis and Applications, vol. 318, no. 1, pp. 43-52, 2006.

[18] B. Halpern, "Fixed points of nonexpanding maps," Bulletin of the American Mathematical Society, vol. 73, pp. 957-961, 1967.

[19] Y. Song and Y. Zheng, "Strong convergence of iteration algorithms for a countable family of nonexpansive mappings," Nonlinear Analysis: Theory, Methods \& Applications, vol. 71, no. 7-8, pp. 30723082, 2009.

[20] J. S. Jung, "Iterative approaches to common fixed points of nonexpansive mappings in Banach spaces," Journal of Mathematical Analysis and Applications, vol. 302, no. 2, pp. 509-520, 2005.

[21] J. G. O'Hara, P. Pillay, and H.-K. Xu, "Iterative approaches to finding nearest common fixed points of nonexpansive mappings in Hilbert spaces," Nonlinear Analysis: Theory, Methods E Applications, vol. 54, no. 8, pp. 1417-1426, 2003.

[22] J. G. O'Hara, P. Pillay, and H.-K. Xu, "Iterative approaches to convex feasibility problems in Banach spaces," Nonlinear Analysis: Theory, Methods E Applications, vol. 64, no. 9, pp. 2022-2042, 2006.

[23] R. Wangkeeree, "An extragradient approximation method for equilibrium problems and fixed point problems of a countable family of nonexpansive mappings," Fixed Point Theory and Applications, vol. 2008, Article ID 134148, 17 pages, 2008.

[24] R. Wangkeeree and U. Kamraksa, "A general iterative method for solving the variational inequality problem and fixed point problem of an infinite family of nonexpansive mappings in Hilbert spaces," Fixed Point Theory and Applications, vol. 2009, Article ID 369215, 23 pages, 2009.

[25] R. Wangkeeree, N. Petrot, and R. Wangkeeree, "The general iterative methods for nonexpansive mappings in Banach spaces," Journal of Global Optimization. In press.

[26] W. Takahashi, Nonlinear Functional Analysis: Fixed Point Theory and Its Applications, Yokohama Publishers, Yokohama, Japan, 2000.

[27] F. E. Browder, "Convergence theorems for sequences of nonlinear operators in Banach spaces," Mathematische Zeitschrift, vol. 100, pp. 201-225, 1967.

[28] T.-C. Lim and H.-K. Xu, "Fixed point theorems for asymptotically nonexpansive mappings," Nonlinear Analysis: Theory, Methods E Applications, vol. 22, no. 11, pp. 1345-1355, 1994.

[29] J.-W. Peng and J.-C. Yao, "A viscosity approximation scheme for system of equilibrium problems, nonexpansive mappings and monotone mappings," Nonlinear Analysis: Theory, Methods E Applications, vol. 71, no. 12, pp. 6001-6010, 2009.

[30] K. Eshita and W. Takahashi, "Approximating zero points of accretive operators in general Banach spaces," JP Journal of Fixed Point Theory and Applications, vol. 2, no. 2, pp. 105-116, 2007.

[31] E. Blum and W. Oettli, "From optimization and variational inequalities to equilibrium problems," The Mathematics Student, vol. 63, no. 1-4, pp. 123-145, 1994.

[32] P. L. Combettes and S. A. Hirstoaga, "Equilibrium programming in Hilbert spaces," Journal of Nonlinear and Convex Analysis, vol. 6, no. 1, pp. 117-136, 2005. 